\title{
Adapting a health systems strengthening model to improve access to health services in a factory: A pilot project in Haiti
}

\author{
Carolyn Rodehau \\ Population Council \\ David Wofford \\ Population Council \\ Suzanne Brockman
}

Follow this and additional works at: https://knowledgecommons.popcouncil.org/departments_sbsr-rh

Part of the Demography, Population, and Ecology Commons, Family, Life Course, and Society Commons, and the International Public Health Commons How does access to this work benefit you? Let us know!

\section{Recommended Citation}

Rodehau, Carolyn, David Wofford, and Suzanne Brockman. 2016. "Adapting a health systems strengthening model to improve access to health services in a factory: A pilot project in Haiti," brief. Washington, DC: Population Council, The Evidence Project. 



\section{BACKGROUND}

Haiti has a fragile healthcare system and a shortage of skilled health workers, many of whom emigrated after the 2010 earthquake (Better Work Haiti 2013a). Close to half of the population lacks access to formal healthcare services.

The apparel industry is one of the largest employers in Haiti, with 30,000 workers, 66 percent of whom are women (Better Work Haiti 2015). Under the Haitian labor code, apparel factories are required to hire a number of nurses and doctors according to the size of their workforce and to meet related occupational health and safety regulations. Workplace health facilities have the potential to fill gaps in the Haitian health system by offering health services to a sizable proportion of the population that would otherwise lack access to health care. However, compliance with workplace health regulations is poor, as is the performance of factory health care staff. For example, 91 percent of factories had less than the number of legally required onsite medical staff in 2013 (Better Work Haiti 2013b).

There are many barriers to healthcare access in Haiti. Hours of operation for public sector services are not compatible with the long hours of most low-wage factory workers. Private sector services are often unaffordable. In Haiti, as in other low- and middle-income countries, the workplace clinic is an access point to reach workers, especially women workers, with health information, products, and services. In fact, recognizing that the workplace has the infrastructure to provide health information and services to a large number of beneficiaries, the World Health Organization has established the workplace as a priority setting for health promotion and health education and has adopted a platform - the Healthy Workplace Model - calling for a comprehensive approach to workplace health (WHO n.d.).

Despite the opportunity, in Haiti and elsewhere, worker health and workplace health functions are a low priority for companies, and services are limited to occupational health and safety considerations such as first aid and personal protective equipment. Little attention is given to the quality of health services and the capacity of health providers to offer basic primary care, family planning and reproductive health services.

Companies tend to view their onsite infirmaries, personnel, and services as costly requirements rather than an integral part of their business operations. Line supervisors take little interest in the health of their workers, and discourage workers from seeking care, onsite or offsite, unless it is an emergency. The health staff lack direction and are underutilized. As a result, the quality of services is often poor. It is not uncommon to find practices below standard and that put patient safety at risk.

Little attention is given to the quality of health services and the capacity of health providers to offer basic primary care, family planning and reproductive health needs.

In 2013, the RAISE Health Initiative** partnered with an American-owned garment factory in Port-au-Prince, Haiti on a pilot project to improve workplace health functions by adapting a health systems strengthening (HSS) approach. At the time of the project, the factory employed 1,600 workers, 60 percent of whom were women. The factory had an onsite infirmary, with services provided by five nurses (one less than required by Haitian law for a factory of that size), and two part-time doctors. It also had three health stations in the production halls, each staffed by a nurse. The factory was committed to worker health and reproductive health, but faced challenges managing its health functions.

This pilot project sought to demonstrate the value of a new management approach to workplace health services and practices in a garment factory. Specifically, the pilot looked at how the HSS model could be adapted to the private sector and help build the factory's capacity to manage the health staff and infirmary, institute clinical best practices, and expand the primary care roles for nurses, including providing family planning and reproductive health services. It aimed to show that better management of worker health would benefit the factory through effective use of existing resources, improved human resources processes, and better productivity.

\section{A HEALTH SYSTEMS STRENGTHENING APPROACH}

At the start of the pilot, the RAISE Health team conducted a needs assessment of the factory's clinical services - including supplies and equipment, service quality, record keeping, and management and supervision - through semi-structured interviews, document reviews, observations, and focus groups. Based on the assessment, the team identified areas to improve the factory's health functions in each of the six core elements of HSS:

**The factory pilot was initiated under the USAID-funded Evidence to Action Project and later transitioned to the Evidence Project, USAID's flagship project for strengthening family planning and reproductive health through implementation science. Critical financial support for the pilot was also provided through a grant from the HRA Pharma Foundation. 
1. Human Resources for Health: The nurses' knowledge of primary care and reproductive health issues was limited, and there wasn't a system to develop their knowledge and skills. The nurses did some health education with workers, but needed training on participatory education techniques, interactive outreach strategies, and where to find up-to-date health education materials.

2. Health Finance: The factory paid for five nurses, two part-time doctors, and health supplies, but did not make full use of these resources. There was a need for more systematic budgeting of these expenditures and linking them to performance and planning goals.

3. Health Governance: The lines of responsibility and accountability were unclear at the management and infirmary levels. Health services were not well integrated into business functions; in particular, there was not a proactive supervisory structure for the nurses. Furthermore, policies and practices for the recruitment, hiring, and evaluation of nurses and for job descriptions and performance goals were inadequate.

4. Health Information: The factory did not collect or use health-related data in a way that enabled it to respond to worker health needs, plan education activities, or improve performance. Infirmary record-keeping, file management, and data collection processes were not standardized.

\section{Medical Products, Vaccines, and Technologies: The} workplace infirmary stocked some family planning methods, which were offered free of charge, but did not have a process or clear accountability for managing inventory. Stock outs and delays getting some family planning methods were common.

6. Service Delivery: The infirmary lacked the processes, protocols and basic practices necessary to provide high quality care related to hygiene, patient histories, and health information. Additionally, the layout and location of the infirmary put patient privacy and confidentially at risk.

\section{INTERVENTION}

The pilot emphasized collaboration, and interventions were developed collectively by a project team comprised of the RAISE Health team, factory management (including the VP of Compliance and the Human Resources Manager), and nurses. The RAISE Health team and the VP of Compliance developed an initial action plan, focused on the six HSS elements, to integrate health into existing management functions.

Over 15 months, RAISE Health conducted five 4-day intensive, participatory trainings with nurses and management

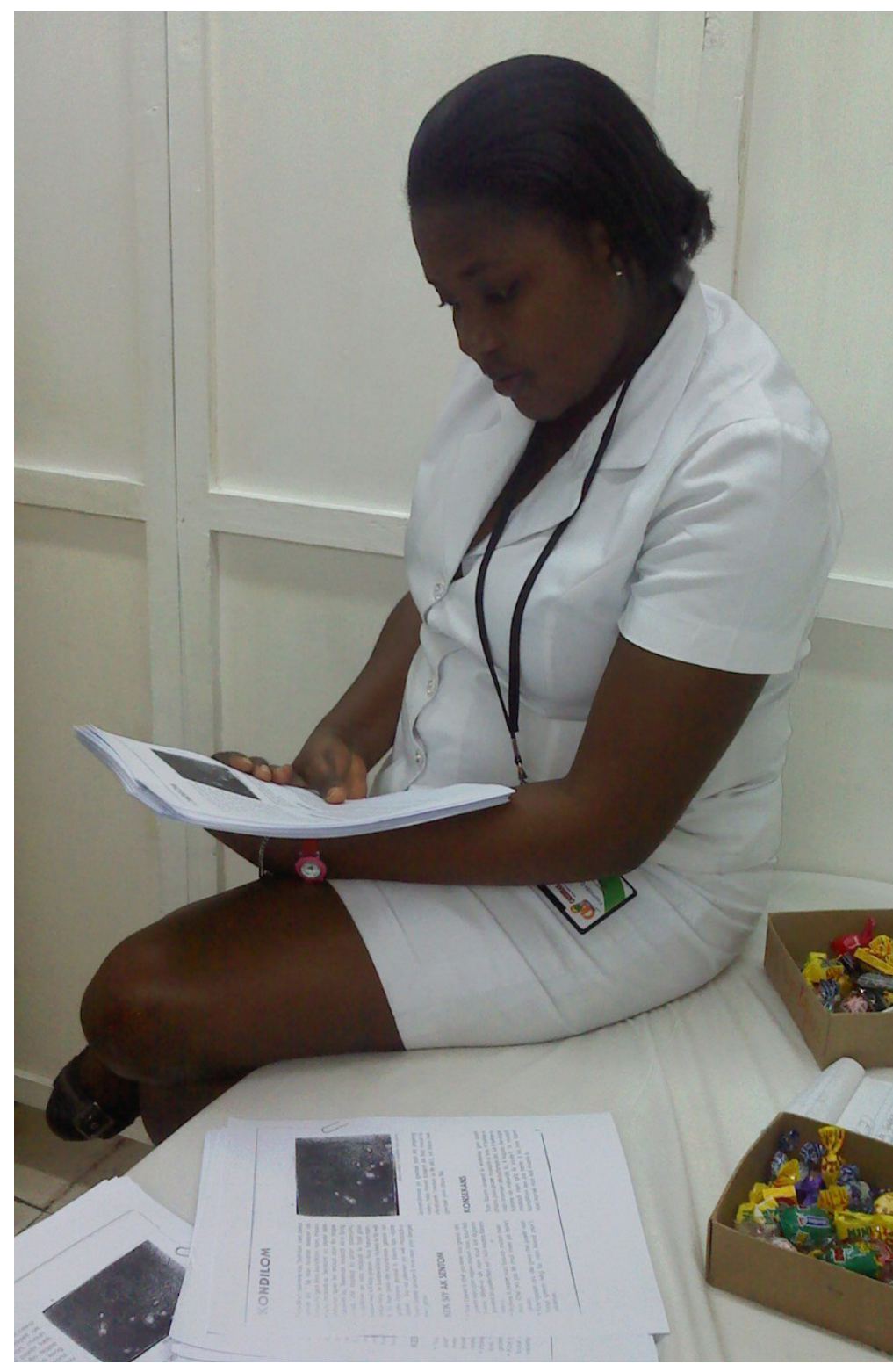

(some training sessions were held with nurses and management together, and some were held separately) to build their respective capacities, define responsibilities, and establish practices and processes. In between the onsite trainings, RAISE Health provided ongoing, remote support via email and monthly Skype calls. This structure promoted factory ownership of the process and sustainability of the changes.

Several tools were adapted and applied to strengthen processes and practices, including:

- The Population Council's Family Planning Balanced Counseling Strategy Plus (BCS+).

- John Snow International's Contraception Inventory and Supply Chain Management tools.

- A new health education approach, based on the Participatory Rural Appraisal (PRA) trainings and other tools. 
- New protocols for medical records, documentation forms, infection control, privacy and confidentiality, adapted from materials used in Federally Qualified Health Centers in the United States.

- A new process for hiring nurses based on job descriptions, qualifications, and formal interviews.

- A revised spreadsheet and process for tracking worker absenteeism.
The nurses are now responsible for developing education activities and worker outreach. This includes weekly "walkabouts" to share basic health messages with workers on the production lines, distributing condoms, and holding health education sessions at lunchtime. Nurses reported that they enjoyed this new active role, and data suggests that more workers were accessing family planning at the end of the project than when it began.

Monthly data was collected on health education and reproductive health indicators to monitor project processes, and interviews with workers and staff helped indicate changes over time.

\section{RESULTS}

The pilot showed that adapting an HSS model to a factory setting improved the factory's health care services, including family planning, clinical care practices and standards, the capacity of its nursing staff and management team, and the use of data. Specifically:

Factory management developed a stronger, more integrated approach to managing health services. The project established new management

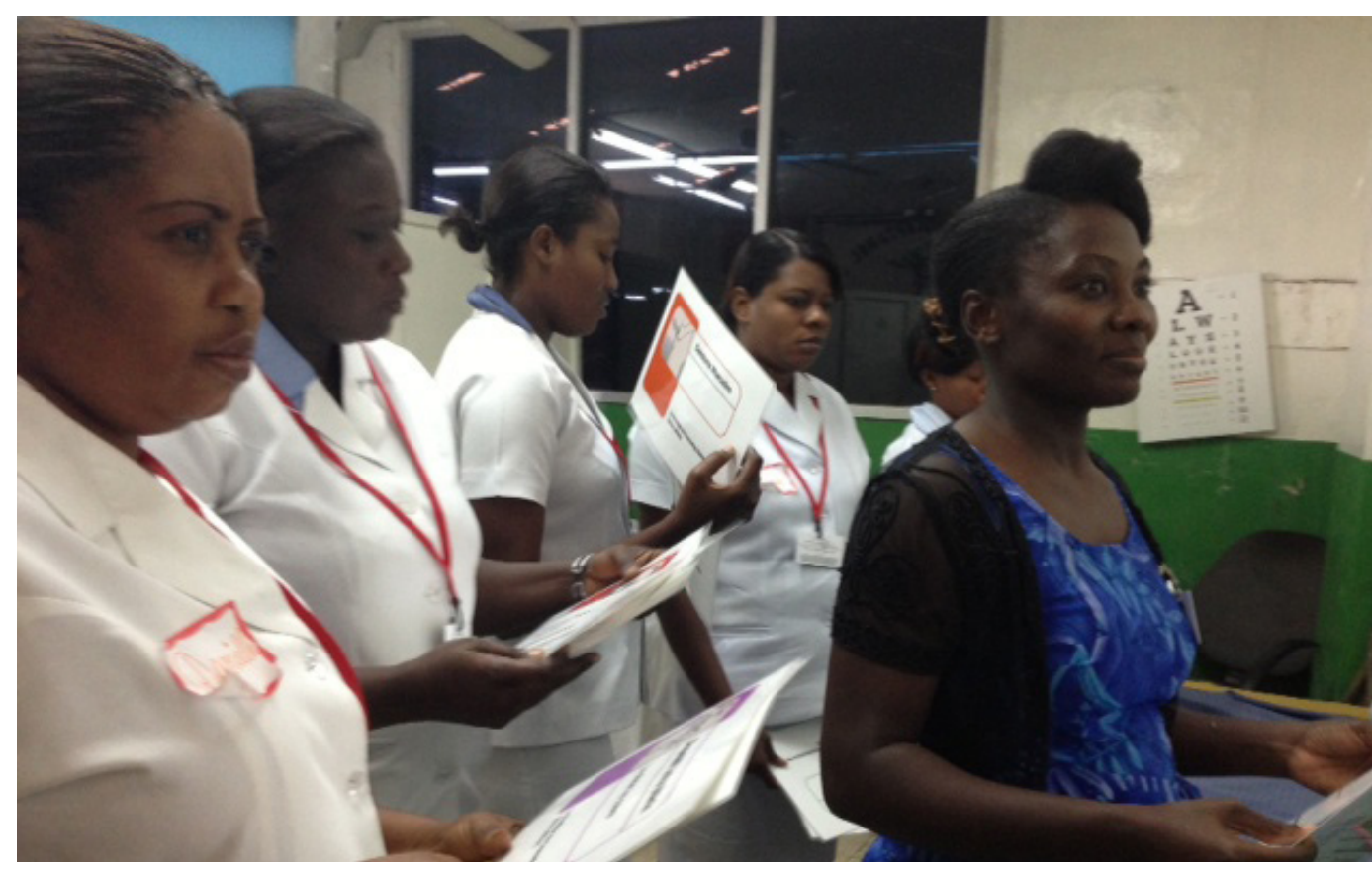
roles and lines of responsibility for health services that have continued past the end of the pilot. A designated middle manager - the Head of Human Resources - now provides supportive supervision to health staff and oversees infirmary practices and educational activities. A supervisory structure of weekly and monthly meetings, data collection, and action planning is still in use. A formal hiring process was established for nurses and other positions, and a human resources process was established to follow up with absent workers to see if their absence was health related and required an infirmary visit.

Introducing quality clinical practices and standard operating procedures improved operations and care. The factory rebuilt its infirmary to ensure patient privacy and new protocols were instituted for record-keeping, data management, and handling hazardous medical waste. The inventory management system improved the supply of contraceptives available at the infirmary. The factory also established a new process in which nurses prepared patient charts and took vital signs to more efficiently use doctors' time.

Nurses' capacity for education and worker outreach was expanded, resulting in increased use of services.

\section{LESSONS LEARNED BUILD ON EXISTING PROCESSES AND STRUCTURES}

A key principle for the pilot project was to build on structures that already existed. While RAISE Health did create some new processes, these took into account existing processes and systems. For instance, the factory and health facility already collected some data; this project mainly added to or improved the existing data collection systems. The team formalized and defined reporting relationships to enable greater accountability and greater autonomy at all levels.

\section{FOCUS ON PERSONNEL AND MANAGEMENT PRACTICES}

It is very important to hire qualified people and define responsibilities. The new hiring process helped factory management avoid nepotism (which had previously been common) and find qualified, committed employees. Establishing management processes - regular meetings, action planning, checklists, and job descriptions - improved accountability and enabled capable staff to do more. These processes helped management to assess performance of nurses and staff, as well as identify who to hire and who to promote. 
A key management change was making the Human Resources Manager responsible for the infirmary and for ensuring all related policies and processes were followed. The defined roles and clear supervisory functions enabled the VP of Compliance to manage the process rather than perform all the tasks.

\section{RECOGNIZE COMPETING PRIORITIES}

A common objection from line supervisors and managers was that health services and health education activities take valuable time away from production. The establishment of a patient management system in the infirmary responded both to a clinical need for improved operations (increased privacy) and the production needs of workers (by reducing waiting time to see a doctor).

\section{BUY-IN OF MIDDLE MANAGEMENT/LINE SUPERVISORS IS CRITICAL}

The VP of Compliance and the Human Resources Manager handled the engagement of middle managers and line supervisors, who have the most direct contact with workers, which was more effective than if engagement had been led by the RAISE Health team. However, RAISE Health should have provided the VP more support from the start in briefing middle management and line supervisors on the project goals and the connection between worker health and productivity.

\section{STRENGTHEN PROBLEM-SOLVING SKILLS}

Nurses were not empowered to make decisions or take action to solve work flow or clinical operation issues. There was a strong culture of hierarchy, a belief that one needed permission from supervisors to take action, and a tendency to view responsibilities in narrow terms. For example, the Chikungunya epidemic quickly caused the infirmary waiting area to overflow with workers. The nurses and Human Resources Manager had successfully created a patient management system to limit the waiting time for each worker with non-emergency needs and avoid lost time at work. Yet, when the waiting room filled up, no one took responsibility for the situation or viewed the problem as solvable. This issue was solved once the cause was identified (workers coming to the clinic without reporting to work) and clinic staff were provided training on problem-solving.

\section{A WAY FORWARD}

This pilot project demonstrated that adapting an HSS model can successfully improve a factory's health care services, including family planning, clinical care practices and standards, as well as the capacity of its nursing staff and management team, with changes maintained after implementation. The results also suggest that an important part of the business case for workplaces to invest in employee health is the value of better managing existing but under-used resources. In their daily routines, workplace health providers can do much more to educate workers, promote healthier behaviors, and provide needed services or refer workers to quality services in the community at a relatively low cost to business. Finally, it provides further evidence that workplace clinics should be required to meet basic standards and apply good health practices - adapted to the workplace context - just as primary care clinics do. This has implications for practitioners and policymakers who may consider including commercial workplaces in their efforts to strengthen health systems and expand access to general and reproductive health services.

In their daily routines, workplace health providers can do much more to educate workers, promote healthier behaviors, and provide needed services or refer workers to quality services in the community at a relatively low cost to business.

Through the pilot, RAISE Health achieved important results and broader impact, beyond the results at the factory, including:

- This model was replicated by Levi Strauss \& Co. as part of its Worker Well-being Initiative at a factory in Egypt. The Levi Strauss Foundation, with the Evidence Project, has developed a publicly available toolkit, "Managing Health at the Workplace: A Guidebook," using the tools, processes, and practices developed under the Haiti pilot and in Egypt, to assist its supplier factories adopt better management practices for worker health.

- RAISE Health partnered with Business for Social Responsibility (BSR) to develop and test "Workplace Health Facility Guidelines and Management Benchmarks." These materials were tested in BSR HERproject factories in Bangladesh and made publicly available in 2015. These guidelines are being used in other HERproject locations and are expected to be tested and adapted in other contexts, such as India and the Philippines.

In addition, through the Evidence Project, these products and the experience gained in Haiti are being used to influence global corporate standards and policies for supply chain factories and farms. This work is also informing a USAID project in Cambodia, the Cambodia Worker Health Coalition, led by the Evidence Project and Marie Stopes International Cambodia. 


\section{REFERENCES}

Better Work Haiti. (2013a). "Better Work Haiti: Garment Industry 7th Biannual Synthesis Report under the HOPE II Legislation." International Finance Corporation, Geneva: ILO.

Better Work Haiti. (2013b). "Better Work Haiti: Garment Industry 7th Biannual Synthesis Report under the HOPE II Legislation." International Finance Corporation, Geneva: ILO.

Better Work Haiti. (2015). “Better Work Haiti: Garment Industry 10th Biannual Synthesis Report under the HOPE II Legislation." International Finance Corporation, Geneva: ILO.

WHO. Occupational Health: Workplace Health Promotion

\section{THE EVIDENCE PROJECT}

Population Council 4301 Connecticut Avenue NW

Suite 280

Washington, DC 20008 USA

tel +12022379400

evidenceproject@popcouncil.org

\section{CONTRIBUTORS}

Carolyn Rodehau David Wofford

Suzanne Brockman
(3) USAID

The Evidence Project is made possible by the generous support of the American people through the United States Agency for International Development (USAID) under the terms of cooperative agreement no. AID-OAA-A-13-00087. The contents of this document are the sole responsibility of the Evidence Project and Population Council and do not necessarily reflect the views of USAID or the United States Government.

\section{Evidence}

The Evidence Project uses implementation science-the strategic generation, translation, and use of evidence-to strengthen and scale up family planning and reproductive health programs to reduce unintended pregnancies worldwide. The Evidence Project is led by the Population Council in partnership with INDEPTH Network, International Planned Parenthood Federation, PATH, Population Reference Bureau, and a University Research Network.

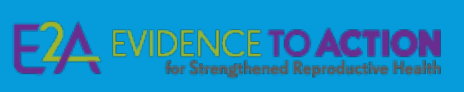

The Evidence To Action (E2A) Project is USAID's global flagship for strengthening family planning and reproductive health service delivery. The project aims to address the reproductive healthcare needs of girls, women, and underserved communities around the world by increasing support, building evidence, and leading the scale-up of best practices that improve family planning services.

\section{Meridian Crouplaternational, Inc.}

Meridian Group International, Inc. is a woman-owned, small busivative programs and partnerships that benefit both business and society. As an Evidence Project partner, Meridian combines implementation science with its extensive experience implementing workplace health programs and promoting better policies and practices.

(c) 2016 The Population Council, Inc.

Suggested Citation: "Adapting a Health Systems Strengthening Model to Improve Access to Health Services in a Factory: A Pilot Project in Haiti.” Brief. Washington, DC: Population Council, Evidence Project. 